\title{
Lecturas de la oposición al aborto
}

\section{Patricia Varela $^{1}$}

\section{Introducción}

Cuando se habla del aborto el debate se centra en el inicio de la vida, en la necesidad de proteger al que está por nacer, en la moral y en los valores. A nadie le importa cómo, cuándo y por qué una mujer abortó o desea abortar. Qué poderosas razones tiene para decidir, a pesar de todos los riesgos que implica, someterse a una intervención así. Nadie se formula la pregunta sobre qué demandas insatisfechas esconden las cifras de aborto: demandas por educación sexual, por anticoncepción, por cambios culturales que permitan efectivamente ejercer el derecho a la sexualidad y a la maternidad (Lagos, 2001, 7).

Si nadie se pregunta sobre los motivos que mueven a una mujer a practicarse un aborto, mucho menos alguien se pregunta por las verdaderas lógicas que operan detrás de la negación de los derechos sexuales y los derechos reproductivos de las mujeres. ¿Cuáles son las lógicas de dominación que operan detrás de la penalización del aborto?, ¿qué es lo que se produce y reproduce a través del control del cuerpo de las mujeres?, ¿qué es lo que se esconde detrás del mandato de maternidad obligatoria?

La penalización absoluta del aborto es ajena a la tradición jurídica chilena. Antes de la promulgación del primer Código Sanitario en 1931, la práctica del aborto para resguardar la salud y la vida de la mujer era considerada lícita. La normativa sanitaria de 1931 sólo cumplió la función de positivar una práctica jurídicamente aceptable y regular su realización (APROFA y RSMLC, 2008, 22).

Entre 1931 y 1989, el artículo 119 del Código Sanitario regulaba el aborto terapéutico en los siguientes términos: Sólo con fines 
terapéuticos se podrá interrumpir un embarazo. Para proceder a estas intervenciones se requiere la opinión documentada de dos médicos-cirujanos (APROFA y RSMLC, 2008, 17). ${ }^{2}$

El aborto terapéutico era considerado lícito, pues estaba plenamente justificado en aras a evitar un mal inminente.

Actualmente la legislación sanitaria, en lo referido al aborto, difiere completamente de la que rigió en Chile durante gran parte del siglo XX. El Código Sanitario regula el aborto en los siguientes términos: No podrá ejecutarse ninguna acción destinada a provocar el aborto (Artículo 119). Esta nueva redacción data de 1989, momento en el cual la dictadura militar eliminó por medio de la Ley 18.826 la institución del aborto terapéutico. Con esta modificación el aborto quedó absolutamente prohibido, tanto en la legislación sanitaria como en la penal (APROFA y RSMLC, 2008, 21).

Junto con este cambio legislativo, la vida y la salud de las mujeres salió de la discusión para dar paso a discusiones sin salida tales como el inicio de la vida y los derechos humanos del que está por nacer. De un momento a otro el embarazo dejó de ocurrir en el cuerpo de una mujer, de una mujer con historia, con sueños, con aspiraciones, con temores, con proyectos.

Cabe entonces preguntarse, ¿qué es lo que se resguarda con la penalización del aborto?, ¿qué es lo que realmente importa a las personas e instituciones que se oponen al aborto? Queda claro que lo que importa no es ni la vida ni la salud de las mujeres. También está claro que lo que importa realmente no es la vida del potencial niño o niña, pues quienes abogan por su derecho a la vida nunca se preocuparán de sus condiciones de existencia una vez que haya nacido. Entonces, ¿cuáles son los reales intereses que se esconden detrás de la oposición al aborto?

En el presente texto se intentarán realizar algunas posibles lecturas de la oposición al aborto desde la Teoría de Género y las Ciencias Sociales. A través de la lectura y el análisis de una serie de corrientes y de autores y autoras intentaremos dilucidar las bases teóricas y conceptuales de esta oposición, las cuales operan a través de la voz de una serie de actores, que en Chile son principalmente la Iglesia Católica y los sectores políticos de la derecha conservadora. 


\section{Las posibles lecturas de la oposición al aborto desde la Teo- ría de Género y las Ciencias Sociales}

\section{1- La lectura desde el psicoanálisis}

\section{a) De Freud a Lacan: El Complejo de Edipo y el Complejo de Castración}

Sigmund Freud acuña la noción de "Complejo de Edipo", para denominar el proceso por el cual tanto hombres como mujeres deben pasar y resolver para constituirse como sujetos.

Para Freud el bebé, sea niño o niña, es un sujeto activo de deseo, y su objeto de deseo es su madre, que hasta ese momento lo es todo. Pero, esta situación se quiebra cuando entra en escena el padre y le hace saber al hijo varón que su madre es propiedad de él y que en el futuro el tendrá su propia mujer, distinta de su madre. El niño entonces, frente a la autoridad del padre, y temiendo ser castrado (complejo de castración) ${ }^{3}$ decide obedecer, comienza a separarse de su madre y su padre se transforma en su referente, dando paso así a la constitución del niño en sujeto masculino.

En el caso de la niña, ella también desea a su madre, pero llega un momento en que se da cuenta que ella no tiene pene, y que además los cuerpos sin pene tienen menos valor. Entonces ella se aleja de su madre por dos motivos: porque la desvaloriza porque no tiene pene y porque la culpa de no haberle dado pene o de habérselo cortado. En ese momento la madre deja de ser su objeto de deseo y se "abyecta", lo que provoca a la niña una depresión, pues en el fondo sabe que ella es como su madre. Entonces, la niña se acerca al padre, en un principio porque piensa que él le puede dar un pene, y luego, cuando asume que esto no ocurrirá, la imagen del padre, en tanto imagen masculina, se vuelve su objeto de deseo porque desea que él le dé un hijo. Y este punto es fundamental, pues para la teoría freudiana el hijo equivale simbólicamente al pene, lo que le lleva a suponer que toda mujer desea un hijo en tanto desea poseer pene. En palabras del propio Freud:

El complejo de Edipo de la niña es mucho más unívoco que el del niño (...) La renuncia al pene no es soportada sin la tentativa de una compensación. La niña pasa podríamos decir que 
siguiendo una comparación simbólica de la idea del pene a la idea del niño. Su complejo de Edipo culmina en el deseo, retenido durante mucho tiempo, de recibir del padre, como regalo, un niño, tener de él un hijo. Experimentamos la impresión de que el complejo de Edipo es abandonado luego lentamente, porque este deseo no llega jamás a cumplirse. Los dos deseos, el de poseer un pene y el de tener un hijo perduran en lo inconsciente intensamente cargados y ayuda a preparar a la criatura femenina para su ulterior papel sexual. ${ }^{4}$

De este modo, para Freud existen tres destinos distintos de la feminidad:

1.- La maternidad, que representa el destino "normal" pues es la superación de la envidia del pene y la realización a través del ser madre en tanto el hijo es el equivalente simbólico del pene que no se posee.

2.- Quedarse en la envidia del pene, que es el destino anormal que habríamos tomado, entre otras, las feministas.

3.- La renuncia a la sexualidad, que también es un destino anormal, y que es el destino, por ejemplo, de las místicas.

Se observa entonces que, para el psicoanálisis freudiano, la maternidad es el destino natural o "normal" de todas las mujeres, y que sólo las mujeres que desean la maternidad se habrían constituido correctamente como sujetos en tanto habrían superado su complejo de Edipo.

En esta misma línea del psicoanálisis surge Jacques Lacan, quien continúa el trabajo de Freud aunque con algunas diferencias. Lacan diferencia tres órdenes por los cuales transita el sujeto: el orden real, el orden imaginario, y el orden simbólico. El orden imaginario es el paso necesario para la coagulación del yo, es el paso por el estadio del espejo, momento en el cual el niño (de entre 6 y 18 meses) se mira en el espejo y se reconoce, es la identificación, el momento en que el sujeto asume una imagen como la imagen propia, es "la matriz simbólica en la que el yo (je) se precipita en una forma primordial, antes de objetivarse en la dialéctica de la identificación con el otro y antes de que el lenguaje le restituya en lo universal su función de sujeto" (Lacan, 1989, 87). 
Pero este orden imaginario es un espacio de sometimiento a la imagen de perfección del padre, y para que el sujeto pueda alcanzar el orden siguiente, el simbólico, y lograr constituirse en yo, deberá separarse definitivamente de la madre, es decir, sólo una vez que complete el asesinato de la imagen materna, el marricidio simbólico, es posible que culmine la coagulación del yo.

La construcción realizada por el psicoanálisis freudiano y lacaniano respecto a la maternidad es al menos perversa, cargada de una tremenda violencia simbólica. En un primer momento se impone a las mujeres la maternidad como la única forma exitosa de superación del Edipo, para luego obligarnos a matar a la madre como condición necesaria para nuestra constitución como sujetas.

Como primer punto, el psicoanálisis da a las mujeres la maternidad como única alternativa viable y normal para la realización personal, reafirmando y entregando argumentos "psíqui$\cos ^{\prime \prime}$ y "médicos" al mandato de maternidad obligatoria. Poniendo a las mujeres que optan por caminos distintos a la maternidad en el plano de lo anormal, de la no resolución satisfactoria de sus procesos de desarrollo psíquico y sexual.

Pero lo curioso es que, mientras se ensalza la maternidad como el único camino normal de la feminidad se postula que para lograr la constitución como sujeto la madre se debe matar simbólicamente. Es decir, se reduce la mujer a la madre y luego se la mata. La madre nos sirve en el primer momento en tanto espacio para la vida (embarazo), luego porque nos proporciona lo necesario para vivir (alimento, abrigo, etc.), pero una vez cumplidas esas funciones la madre no sólo es prescindible, sino que debe anularse. La madre, y por tanto la mujer, pues su única forma de realizarse como sujeta es la maternidad, es reducida a instrumento reproductivo capaz de dar vida, incubar y amamantar. Luego de eso es un estorbo para la constitución del yo.

La negación del aborto es también la reducción de la mujer a su capacidad reproductiva, negándole su historia, sus deseos, su vida, sus derechos. Es erigir la maternidad como el único rol y destino válido y legitimo para una mujer. No importa que el 
embarazo y la maternidad coarten proyectos personales, no importa que no lo queramos, pues es el destino de toda mujer. ¿Qué tan terrible puede ser obligar a una mujer a seguir con un embarazo y parir si en el fondo, a nivel inconciente todas lo deseamos?

\section{b) Julia Kristeva y la paradoja de la maternidad}

Julia Kristeva en el Stabat Mater plantea la paradoja que existe en numerosas civilizaciones, pero en especial en las sociedades cristianas con respecto a la figura de la madre, ya que por un lado la condición femenina es absorbida por la maternidad, siendo esta ensalzada y venerada a través del culto a la virgen María, pero por otro, la maternidad ensalzada por el cristianismo es una fantasía, pues se trata de una madre arcaica idealizada.

Sólo en el patriarcado cristiano se ha llevado al extremo la fetichización de sustituir mujer por madre, pero al hacer esta sustitución se descorporiza el cuerpo de la mujer, porque la madre idealizada es una madre sin cuerpo, sin sexualidad, es la María siempre virgen y sin pecado concebida, es María convertida en ÚNICA por los dogmas cristianos, única en su sexo, capaz de concebir y parir siendo virgen, capaz de ser concebida sin pecado.

La autora señala además que lo maternal virginal, representado en la imagen de la virgen María y erigido como ideal de feminidad, es una hábil construcción de equilibrio de concesiones y coacciones a la paranoia femenina. Le da poder a la mujermadre a través de la imagen de María, erigida en reina, transformada en patrona, adorada y venerada, pero a la vez se lo quita, la arrodilla ante el hijo, la vuelve sumisa ante el Padre, "hágase en mi tu voluntad".

Pero este equilibrio esta tambaleándose, y frente a esto Kristeva se pregunta: “ ¿a qué aspectos del psiquismo femenino no da respuesta esta representación de lo maternal o bien, sólo da una respuesta que las mujeres del siglo XX consideran demasiado coercitiva?" (Kristeva, 1987, 228). 
Para Kristeva el problema está en lo no dicho. El primer no dicho es el estallido que ocurre en el cuerpo de la mujer con el embarazo y la llegada del hijo, pues nada se dice sobre el sufrimiento corporal y físico del parto, y sobre todo de la abnegación que implica el hacerse anónima para criar y transmitir al nuevo hijo la norma social, con la que incluso se puede estar en desacuerdo. Es sufrimiento revestido de júbilo. En otras palabras, estos silencios no son más que el silenciamiento de la mujer real, el silenciamiento del cuerpo, de los sufrimientos y sacrificios que implica la maternidad.

No se pare con dolor, se pare el dolor: el niño lo representa y el dolor se instala permanentemente desde entonces. Por supuesto, pueden ustedes cerrar los ojos, taparse los oídos, hacer cursos, carreras, arreglar la casa, pensar en los objetos, en los sujetos. Pero una madre siempre está marcada por el dolor, sucumbe a él. "Y a ti, una espada te atravesará el alma..." (Kristeva, 1987, 214-215).

Kristeva comprende que esta sacralización de la maternidad a expensas de las mujeres de carne y hueso, así como también el rol que juega el culto mariano en tanto generador de equilibrio y estabilidad para el sistema patriarcal, ha generado dos posturas en las mujeres: la aceptación de este orden tradicional sin mayor cuestionamiento, que ha sido y es la postura de la mayoría de las mujeres, y en oposición la negación o el rechazo de la maternidad, que ha sido la postura de ciertos sectores del feminismo.

Pero a Kristeva le interesa matizar este rechazo a la maternidad de algunos feminismos. El problema no es la maternidad en sí misma, el problema es la sacralización que se hace de ella en el cristianismo. Si se compara al cristianismo de la Contrarreforma con las sociedades capitalistas protestantes, éstas últimas reniegan del culto a la virgen, no existe en ellas el culto mariano, y en estas sociedades las mujeres han tenido mayores espacios para la participación social, incluso han vuelto a ser parte del rito, pues estas iglesias reconocen en las mujeres la capacidad de comunicarse con Dios. La Contrarreforma por el contrario es un resurgimiento del feudalismo, más marianismo, menos participación de las mujeres en el mundo público. 
Resulta evidente entonces que en un país como Chile, donde la imagen de la virgen María es venerada y querida por gran parte de la sociedad, el que una mujer rechace la maternidad aparezca como algo al menos extraño. A esto hay que sumar que en nuestro país los poderes fácticos y gran parte del poder político son detentados por sectores ultra católicos, lo que en la práctica se traduce en la imposición del culto mariano, y del ideal femenino procedente de éste, a toda la sociedad. Sólo así se explica que hasta la actualidad el aborto esté penalizado en todas sus formas, pues desde el ideal mariano la mujer sólo debe acatar los designios de Dios, tal como María, "hágase en mi tu voluntad".

Siguiendo a Kristeva es la reducción de la mujer a la maternidad y el culto a la imagen materna de la virgen María lo que ha venido a sustentar y fortalecer, en sociedades predominantemente cristiano-católicas como la nuestra, el mandato de maternidad obligatoria y por tanto la negación de los derechos sexuales y reproductivos de las mujeres. Se espera, y el sistema patriarcalcatólico se asegura de que así sea, que todas las mujeres sigamos el ejemplo de María. Aunque el seguir este ejemplo de maternidad implique la renuncia a nuestra condición de sujetas, aunque implique la renuncia a nuestro derecho a decidir sobre nuestro cuerpo, aunque pueda implicar incluso poner nuestra salud y nuestra vida en peligro.

\section{2- La lectura desde la violencia de género: El cuerpo femenino como medio de expresión del poder patriarcal}

La antropóloga Rita Segato, en sus escritos respecto a la violencia contra las mujeres, ha puesto de relieve la expresividad y la interlocución que lleva implícitas este fenómeno. En el caso de la expresividad y la interlocución Segato plantea que a través de la violación, tortura y muerte de una mujer la hermandad masculina refuerza su poder y su capacidad de control y muerte. El principal mensaje no está dirigido a la mujer asesinada, sino a los pares. En casos como el de Ciudad Juárez, por ejemplo, en el cual los asesinos toman a mujeres desconocidas, a la víctima le es enajenado su cuerpo, para transformarlo en un lienzo que porta un mensaje claro: la reafirmación del poder masculino, la 
desigualdad del orden patriarcal, el no valor del cuerpo femenino, el poder y la impunidad.

Respecto a la territorialidad, la autora plantea que el cuerpo femenino y el territorio, a lo largo de la historia han sido entendidos como un continuo, y sólo basta con pensar en cualquier guerra y las violaciones de niñas y mujeres que implica para comprobar esta tesis. Sin embargo, esta relación entre cuerpo femenino y territorio no sólo implica que el cuerpo de las mujeres sea violado, vejado y torturado para mostrar superioridad sobre el otro masculino (el enemigo) y para simbolizar la conquista territorial, sino que además implica que el cuerpo femenino sea entendido, en palabras de Segato, "como el lugar privilegiado para significar el dominio y la potencia cohesiva de una colectividad". Y es en este último punto donde quiero quedarme.

Por un momento dejemos de pensar en el feminicidio y la violencia física y pensemos en las violencias cotidianas y naturalizadas a las que somos sometidas las mujeres. Pensemos en el mandato de la maternidad obligatoria, pensemos en el no poder decidir libremente sobre nuestra sexualidad y nuestra capacidad reproductiva, y pensemos en las mujeres que por insistir en su derecho a decidir se someten a abortos inseguros que pueden provocar severas secuelas y hasta la muerte.

Segato define la violación como:

Uso y abuso del cuerpo del otro sin que este participe con intención o voluntad compatibles, la violación se dirige al aniquilamiento de la voluntad de la víctima, cuya reducción es justamente significada por la pérdida del control sobre el comportamiento de su cuerpo y el agenciamiento del mismo por la voluntad del agresor (Segato, 2004, 6).

¿No podríamos acaso definir casi exactamente igual la negación que se hace a las mujeres de sus decisiones reproductivas?, ¿el tener que vivir de manera obligada un embarazo no deseado en el propio cuerpo no es acaso un aniquilamiento de la voluntad y una reducción significada en la pérdida del control sobre el comportamiento del cuerpo? 
La autora sostiene que los agresores emiten mensajes en dos ejes, un eje vertical dirigido a la mujer víctima, donde el mensaje adquiere un carácter punitivo y el agresor se arroga el papel de moralizador, de paladín de la moral social, "porque, en ese imaginario compartido, el destino de la mujer es ser contenida, censurada, disciplinada, reducida, por el acto violento de quien reencarna, por medio de este acto, la función soberana" (Segato, 2004,8). ¿No es acaso la obligación de llevar a termino un embarazo no deseado también una medida disciplinaria y moralizante?, sólo basta hacer alusión a un sinnúmero de frases que con frecuencia son utilizadas en la sociedad e incluso en los servicios de salud sobre todo contra las embarazadas adolescente, "no pensó acaso cuando lo estaba haciendo", "debería entonces haber cerrado las piernas", "no le gustó andar haciendo cosas de grande, entonces apechugue".

El segundo eje, el horizontal, es el eje de interlocución con los pares, es el eje mediante el cual se envía a la fratría, a la hermandad viril, el mensaje de unidad, de poder. La violencia implícita en el mandato de la maternidad obligatoria, la prohibición de la anticoncepción y el aborto, la disminución de la sujeta mujer a un aparato reproductivo, cumple aquí una función muy similar a la que cumplen los feminicidios en Juárez para las mafias patriarcales: enviar a la comunidad (ya sea la comunidad mafiosa, ya sea la comunidad religiosa, ya sea la sociedad en general) un mensaje de poder y control sobre la vida de las mujeres, y sobre todo un mensaje de reafirmación y reproducción del orden patriarcal desigual e injusto.

Y al igual que en Juárez el papel sobre el que se escribe este mensaje es el cuerpo de las mujeres, es el cuerpo de una niña de 15 años obligada a parir el fruto de un incesto, es el cuerpo de la mujer pobladora obligada a tener su sexto hijo/a, es el cuerpo de la mujer que llega al borde de la muerte a un hospital por un aborto clandestino realizado en malas condiciones.

La autora plantea que en el sistema sexo-género vigente, la masculinidad requiere ser primero obtenida y luego reafirmada de manera constante. Y esta obtención y reafirmación se realiza mediante un proceso de probación o conquista que esta 
supeditado al cobro injusto y violento de tributos de otro, que es excluido de la casta masculina.

Esto es así porque en el larguísimo tiempo de la historia del género, tan largo que se confunde con la historia de la especie, la producción de la masculinidad obedece a procesos diferentes a los de la producción de femineidad. Evidencias en una perspectiva transcultural indican que la masculinidad es un status condicionado a su obtención -que debe ser reconfirmada con una cierta regularidad a lo largo de la vida- mediante un proceso de probación o conquista y, sobre todo, supeditado a la exacción de tributos de un otro que, por su posición naturalizada en este orden de status, es percibido como el proveedor del repertorio de gestos que alimentan la virilidad. Ese otro, en el mismo acto en que hace entrega del tributo instaurador, produce su propia exclusión de la casta que consagra (Segato, 2004, 8).

Queda claro que en el patriarcado somos las mujeres las tributarias de la masculinidad. La masculinidad queda otorgada y confirmada en base a la actividad sexual del varón (actividad sexual que debe ser con una mujer), la masculinidad hegemónica se reafirma en base a cuantas mujeres se penetra y a cuantas mujeres se fecunda, el tributo es la entrega de la sexualidad y de la capacidad reproductiva femenina a los varones, entrega que en gran número de casos tiene, a nivel conciente o inconciente, el carácter de obligatoria o al menos de coaccionada. El cuerpo femenino es el instrumento mediante el cual se cobra el tributo. El control y la soberanía de las mujeres sobre sus propios cuerpos aparece entonces para el patriarcado como una grave amenaza, puesto que sin el tributo femenino obligado desaparece la única forma conocida da hacerse y ser miembro de la comunidad masculina dominante.

Finalmente, ¿por qué la muerte de mujeres a causa de abortos clandestinos no es un tema de preocupación? Parece ser que el bienestar y la salud de las mujeres no es un argumento válido para quienes se oponen al aborto. Es más, para quienes se oponen incluso al aborto terapéutico cuando está en riesgo la vida de la madre, lo natural es que la mujer esté dispuesta a dar su vida, y si no quiere hacerlo por su voluntad la mujer debe ser obligada al "heroísmo o el martirio", basta con leer el siguiente extracto de una intervención de Jaime Guzmán, uno de los principales ideólogos de la Dictadura y ferviente católico: 
La madre debe tener al hijo aunque este salga anormal, aunque no lo haya deseado, aunque sea producto de una violación $\mathrm{o}$, aunque de tenerlo, derive en su muerte (...). Hay personas para las cuales el límite entre el heroísmo o el martirio, por una parte, y la falla moral, por otra, se estrecha hasta hacerse imposible. La mayoría de los seres humanos viven gran parte de sus vidas en una amplia zona intermedia que hay entre ambas, pero la Providencia permite, exige o impone muchas veces a un ser humano que ese cerco se estreche y la persona se encuentra obligada a enfrentar una disyuntiva en la cual no queda sino la falla moral, por una parte, o el heroísmo o el martirio, por otra... (Cit. En Lagos, 2001, 11).

Para Segato, “en un régimen de soberanía, algunos están destinados a la muerte para que en su cuerpo el poder soberano grabe su marca; en este sentido, la muerte de estos elegidos para representar el drama de la dominación es una muerte expresiva, no una muerte utilitaria" (Segato, 2004, 7). Parece ser que para el régimen de soberanía del patriarcado la muerte de mujeres por abortos clandestinos son esas muertes expresivas, ellas son las elegidas para enviar, a través de sus cuerpos muertos, a través de sus úteros destrozados, un mensaje de obediencia a las demás mujeres: el mensaje de sumisión a la comunidad masculina, el mensaje de la maternidad obligatoria, el mensaje de renunciar a la propia sexualidad y al control de la capacidad reproductiva. El mensaje de que si no obedeces puedes morir sin que a nadie le importe.

\section{Conclusiones y comentarios finales}

A lo largo de este trabajo, se han revisado una serie de corrientes de las ciencias sociales desde un enfoque de género, intentando aplicar sus aportes al análisis de la oposición al aborto y de la negación de los derechos reproductivos de las mujeres.

Desde la psicología se ha revisado el psicoanálisis en su vertiente freudiana, lacaniana, y luego feminista con Julia Kristeva. Se ha observado como, Freud y Lacan, a través fundamentalmente del Complejo de Edipo y la Envidia del Pene han fortalecido 
y entregado argumentos al mandato de maternidad obligatoria, mostrando la maternidad como único destino normal para las mujeres, y asumiendo que, como consecuencia lógica de la envidia del pene que todas las mujeres tuvimos en la infancia, todas las mujeres, aunque sea de modo inconciente, deseamos ser madres, entendiendo al hijo o hija como sustituto del pene deseado.

Luego, también desde el psicoanálisis, Julia Kristeva nos ha entregado nuevos elementos para el análisis, mostrándonos como el culto a la virgen ha llevado a la reducción de la mujer a la madre, y como el culto mariano ha alejado cada vez más a la madre de la mujer real de carne y hueso. Así, el ideal de madre aparece como inalcanzable, como algo imposible para las mujeres, pero que sin embargo se nos exige e impone.

Finalmente, y desde la antropología, Rita Segato, con sus análisis respecto a los feminicidios en Ciudad Juárez, ha incorporado al fenómeno de la violencia de género una dimensión de expresividad e interlocución, dimensión que hemos aprovechado para complementar nuestro análisis respecto a la oposición al aborto, intentando mostrar cómo la negación de los derechos reproductivos, al igual que los feminicidios o las violaciones, no son más que un mensaje de reafirmación del poder patriarcal, mensaje que usa como lienzo el cuerpo de las mujeres.

La intención de incorporar nuevas lecturas de la oposición al aborto es aportar tanto en el ámbito teórico como en práctica política nuevos elementos para la lucha por el reconocimiento y el ejercicio libre de los derechos sexuales y los derechos reproductivos de las mujeres. Creemos que mientras más claridad exista respecto a las estructuras que operan tras los discursos conservadores y antiderechos podremos atacarlos y deconstruirlos de manera más eficaz y certera.

El mostrar la penalización del aborto y la negación de los derechos reproductivos como una estrategia de reafirmación y reproducción del sistema sexo-género vigente, responde a nuestro interés por avanzar hacia una sociedad más justa y más igualitaria, donde las mujeres puedan ejercer su sexualidad de manera libre y segura. 
Y siguiendo en la línea de Kristeva, nuestra intención en ningún caso es satanizar la maternidad. No queremos reducir la maternidad a patriarcado del mismo modo que el patriarcado redujo lo femenino a la maternidad. Queremos desacralizar la maternidad, transformarla en una experiencia real y no en un mito o idealización. Queremos que la maternidad deje de ser un mandato y sea una opción, una opción que al ser tomada pueda ser vivida sin coacciones, sin fantasmas del deber ser. Que ya no exista una sola forma de ser madre, que la maternidad ya nunca más sea el producto de un descuido, mucho menos un castigo o una maldición, que es como la viven en la actualidad muchas mujeres. Queremos que exista la posibilidad de optar por no ser madres, y que para las que quieran serlo, exista la posibilidad real de vivir la maternidad con dicha, con placer, con libertad y con autonomía.

\section{Notas}

1 Socióloga, estudiante de Magíster en Estudios de Género y Cultura de la Universidad de Chile.

2 El niño ha visto que su madre y las demás mujeres no tienen pene y piensa que se los han cortado.

3 Freud, Sigmund. "La disolución del Complejo de Edipo". Planetalibro, Septiembre, 2008.

4 Guzmán, Jaime, Actas Oficiales de la Comisión Constituyente. Sesión $87^{\circ}$ del 14 de noviembre de 1974.

\section{Bibliografía}

Asociación de Protección de la Familia y la Red de Salud de Mujeres Latinoamericanas y del Caribe (2008). Documento Final de Consenso "Despenalización del aborto en condiciones biopsicosociales calificadas en que se vulneran gravemente los derechos de las mujeres", del Proyecto "Generación de una Agenda Pública para la Despenalización del Aborto por Indicación Médica en Chile: Formación de capacidades para una Acción Sostenible de Instituciones Privadas de Interés Público en Aborto Terapéutico", Santiago de Chile. 
Freud, Sigmund. "La disolución del Complejo de Edipo". Planetalibro Septiembre 2008. http://www.planetalibro.net/ebooks/eam/ ebook_view.php?ebooks_books_id=136

Kristeva, Julia. Stabat Mater. En "Historias de Amor". México: Editorial Siglo XXI, 1987.

Lacan, Jacques. Escritos. 1966. México: Editorial Siglo XXI, 1989.

Lagos, Claudia. Aborto en Chile. El deber de parir. Santiago: LOM Ediciones, Colección Nuevo Periodismo, 2001.

Segato Rita. Territorio, soberanía y crímenes de segundo estado: la escritura en el cuerpo de las mujeres asesinadas en Ciudad Juárez (nueva versión). Serie Antropología, Brasilia, 2004.

http: / / www.scribd.com/doc/6628508 / Rita-Segato-Territorio-Soberani-y-Crimenes-de-Segundo-Estado 